Results 70 patients investigated for suspected PE in an acute teaching hospital during September 2010 were reviewed. Mean age 58 years (median 61), 60\% female. The majority of patients presented with breathlessness (64\%) and pleuritic chest pain (54\%). There was no documentation of clinical probability in $69 \%$ of notes, however $73 \%$ of imaging requests had clinical probability scores recorded. Eight patients (26\%) did not have any risk factors for venous thrombo-embolism. Four patients had CT pulmonary angiogram following an inconclusive perfusion scan. The majority of the patients $(70 \%)$ were weighed prior to prescribing $\mathrm{LMWH}$. Five $(7 \%)$ patients had their weight estimated and $14(20 \%)$ had no documentation of weight. Creatinine clearance was $<30 \mathrm{ml} /$ min in three patients, one patient had their LMWH adjusted accordingly. More than half of patients (53\%) received incorrect dose of LMWH. No LMWH related complication was recorded in any patient.

Conclusion This small cross sectional study has limitations. Larger studies are needed to evaluate the frequency of harm associated with incorrect prescription of $\mathrm{LMWH}$.

\section{P11 ASSESSMENT OF MALIGNANCY IN PATIENTS WITH IDIOPATHIC PULMONARY EMBOLUS: AN AUDIT}

doi:10.1136/thoraxjnl-2011-201054c.11

R Som. East of England Deanery, Cambridge, UK

Background There are 65000 cases of pulmonary embolus (PE) in hospital per year in England and Wales. There is a significant association between idiopathic venous thrombosis and cancer and an increase in risk of diagnosis of cancer within a year of idiopathic venous thrombosis. The British Thoracic Society (BTS) guidelines suggests that all patients who do not have a major risk factor for PE should receive "a combination of careful clinical assessment, routine blood tests and chest radiography" and only when these indicate possibility of malignancy, should further imaging or invasive investigations for malignancy be considered. Our aim was to evaluate these guidelines in a large teaching hospital in England.

Method A retrospective patient-chart review of all patients admitted with pulmonary embolus over 12 months was performed. A patient was excluded if they had a clear major risk factor for developing PE for example, recent pelvic surgery, known malignancy etc. If a patient had no clear risk factor, the documentation during the admission was reviewed to see whether clinicians were complying with BTS guidelines and assessing for malignancy appropriately. A pro-forma was designed to check this, with 1 point being given for every aspect of history/investigation performed in regards to assessing for cancer for example, 1 point awarded if the patient was asked about recent change in bowel habit; 1 point if the patient's serum calcium was checked. An overall score was given for each clinical assessment for malignancy for each patient (out of 14 for men; out of 15 for women).

Results 202 patients with confirmed PE were admitted over 12 months. 39 patients were included in the study. In summary, compliance with BTS guidelines calling for thorough clinical assessment was poor in a number of parameters-patients were not asked if they were suffering from systemic symptoms of malignancy, or assessed for symptoms and signs of common malignancies associated with PE. Conversely, a number of patients were inappropriately referred for further investigation-particularly imaging-for possible malignancy without a documented history or examination pertaining to a specific malignancy.

Conclusion Compliance with the guidelines from the BTS is poor. Adequate histories and examinations for malignancy are not being performed. This suggests that either the guidelines or the clinical practice needs re-evaluation.

\section{Interferon-gamma assays in TB diagnosis P12 ROLE OF INTERFERON GAMMA RELEASE ASSAY (OUANTIFERON-TB GOLD IN TUBE) IN BLOOD IN THE DIAGNOSTIC WORK UP OF ACTIVE TUBERCULOSIS IN A HIGH TB PREVALENCE REGION}

doi:10.1136/thoraxjnl-2011-201054c.12

${ }^{1} \mathrm{~S}$ Kumar, ${ }^{2} \mathrm{D}$ Gupta, ${ }^{2}$ Verma, ${ }^{2} \mathrm{~S} \mathrm{~K}$ Jindal, ${ }^{2} \mathrm{R}$ Agarwal. ${ }^{1}$ Institute of Liver and Biliary Sciences, New Delhi, India; ${ }^{2}$ Post Graduate Medical Education and Research, Chandigarh, India

Objective To study the role of Interferon gamma release assay (IGRA) (QuantiFERON-TB Gold In Tube) in blood in the diagnostic work up of active tuberculosis (TB) in a high TB prevalence region.

Design Prospective, comparative group study.

Setting Subjects presenting to the services of the Pulmonary Medicine Department of a large tertiary care teaching hospital in northern India.

Methods We prospectively enrolled, 30 cases of smear or histopathology proven newly diagnosed tuberculosis (18 pulmonary (PTB) and 12 extra-pulmonary (EPTB)) patients controls along with 30 healthy controls. All cases and controls underwent Tubercular Skin Test (TST) using $0.1 \mathrm{~mL}$ (1 tuberculin units) of purified protein derivative RT23 and IGRA using QuantiFERON-TB-Gold In Tube assay (QFT) in blood. For TST an induration $\geq 10 \mathrm{~mm}$ was taken as positive. OFT testing was performed and interpreted as per manufacturer's (Cellestis) instructions.

Results We studied 30 patients of active tuberculosis (18 PTB and 12 EPTB) and 30 healthy controls (14 men and 13 women, mean age $35.03 \pm 13.23$ years). TST positivity had a sensitivity of $83.33 \%$ and $66.67 \%$ and specificity of $60 \%$ for both categories for the diagnosis of active PTB and EPTB respectively. In contrast OFT positivity had a sensitivity of $61.11 \%$ and $58.33 \%$ and specificity of $50 \%$ for the diagnosis of active PTB and EPTB respectively.

Conclusions In this study the OFT-IGRA had a limited overall usefulness in the diagnosis of active pulmonary and extrapulmonary TB. OFT, thus can neither be taken as rule in nor rule out test in a

Abstract P12 Table 1 Head to head comparison of TST \& QFT positivity in different categories of tuberculosis patients (vs control)

\begin{tabular}{|c|c|c|}
\hline Parameter & $\begin{array}{l}\text { Value obtained for } \\
\text { TST }(95 \% \mathrm{CI})\end{array}$ & $\begin{array}{l}\text { Value obtained for } \\
\text { OFT }(95 \% \mathrm{CI})\end{array}$ \\
\hline \multicolumn{3}{|l|}{ Pulmonary tuberculosis } \\
\hline Sensitivity for positive test & $83.33 \%(0.5858$ to 0.9642$)$ & $61.11 \%(0.3575$ to 0.8270$)$ \\
\hline Specificity for positive test & $60 \%(0.4060$ to 0.7734$)$ & $50 \%(0.3130$ to 0.6870$)$ \\
\hline $\begin{array}{l}\text { Efficiency (correct } \\
\text { classification rate) }\end{array}$ & $68.75 \%(0.5375$ to 0.8134$)$ & $54.17 \%(0.3917$ to 0.6863$)$ \\
\hline $\begin{array}{l}\text { Predictive value of } \\
\text { positive test }\end{array}$ & $55.56 \%(0.3533$ to 0.7452$)$ & $42.31 \%(0.2335$ to 0.6308$)$ \\
\hline $\mathrm{OR}$ & 7.5000 (1.7791 to 31.6170$)$ & $1.5714(0.4793$ to 5.1526$)$ \\
\hline $\begin{array}{l}\text { Predictive value of } \\
\text { negative test }\end{array}$ & $85.71 \%(0.6366$ to 0.9695$)$ & $68.18 \%(0.4513$ to 0.8614$)$ \\
\hline \multicolumn{3}{|l|}{ Extra pulmonary tuberculosis } \\
\hline Sensitivity for positive test & $66.67 \%(0.3489$ to 0.9008$)$ & $58.33 \%(0.2767$ to 0.8483$)$ \\
\hline Specificity for positive test & $60 \%(0.4060$ to 0.7734$)$ & $50 \%(0.3130$ to 0.6870$)$ \\
\hline $\begin{array}{l}\text { Predictive value of a } \\
\text { positive test }\end{array}$ & $40 \%(0.1912$ to 0.6395$)$ & $31.82 \%(0.1386$ to 0.5487$)$ \\
\hline $\begin{array}{l}\text { Efficiency (correct } \\
\text { classification rate) }\end{array}$ & $61.90 \%(0.4564$ to 0.7643$)$ & $52.38 \%(0.3642$ to 0.6800$)$ \\
\hline $\mathrm{OR}$ & $3.0000(0.7361$ to 12.2268$)$ & $1.4000(0.3620$ to 5.4139$)$ \\
\hline $\begin{array}{l}\text { Predictive value of } \\
\text { negative test }\end{array}$ & $81.82 \%(0.5972$ to 0.9481$)$ & $75 \%(0.5090$ to 0.9134$)$ \\
\hline \multicolumn{3}{|l|}{ Any tuberculosis } \\
\hline Sensitivity for positive test & $76.67 \%(0.5772$ to 0.9007$)$ & $60 \%(0.4060$ to 0.7734$)$ \\
\hline
\end{tabular}


high TB prevalence region like India. TST with all its limitation probably will continue to serve a useful purpose. Larger prospective studies are needed in high incidence countries to determine the sensitivity and specificity of IGRAs, their ability to serve as useful rule-out test for active TB.

\section{P13 INTERFERON-GAMMA RELEASE ASSAY (IGRA) CONVERSION, REVERSION AND IMPLICATIONS FOR THE DIAGNOSIS OF LATENT TUBERCULOSIS INFECTION USING A MULTIMODALITY APPROACH: A RETROSPECTIVE, OBSERVATIONAL STUDY WITHIN A CENTRAL LONDON TB CENTRE}

doi:10.1136/thoraxjnl-2011-201054c.13

${ }^{1} \mathrm{M}$ H Almond, ${ }^{1} \mathrm{M}$ O'Donoghue, ${ }^{2} \mathrm{~N}$ Drey, ${ }^{3} \mathrm{~S}$ Seneviratne, ${ }^{4} \mathrm{~A}$ Lalvani, ${ }^{1} \mathrm{M}$ Wickremasinghe, ${ }^{1} 0 \mathrm{M}$ Kon. ${ }^{1}$ Chest and Allergy Department, St. Mary's Hospital, Imperial College NHS Trust, London, UK; ${ }^{2}$ School of Community and Health Sciences, City University, London, UK; ${ }^{3}$ Department of Clinical Immunology, St. Mary's Hospital, Imperial College NHS Trust, London, UK; ${ }^{4}$ Tuberculosis Research Unit, St. Mary's Hospital, Imperial College NHS Trust, London, UK

Introduction and Objectives Accurate diagnosis \& management of latent tuberculosis infection (LTBI) among TB contacts is critical for both the health of infected individuals and prevention of disease transmission. Interferon gamma release assays (IGRAs) measure $\mathrm{T}$ cell release of interferon-gamma following stimulation by antigens not confounded by the BCG vaccination. The current NICE guidelines recommend their use following a positive TST. In addition some centres have moved to a single step IGRA test for LTBI. Our institution adopts a triple investigation approach comprising a chest radiograph (CXR), TST and IGRA on presentation followed by a rescreen if the TST \& IGRA are discordant or if pulmonary contacts are screened prior to 6 weeks. The aim of our study was to evaluate the prevalence of IGRA conversion and reversion in rescreened asymptomatic $\mathrm{TB}$ contacts that attended our centre.

Methods This was a retrospective, observational study carried out at a central London teaching hospital. The study population comprised 593 consecutive, adult TB contacts screened between 1 January 2008 and 31 December 2010. Data were collected through retrospective review of chest radiographs, TST \& IGRA tests.

Results Of 498 asymptomatic TB contacts screened, 460 had both an initial TST and IGRA performed (Abstract P13 figure 1). 81

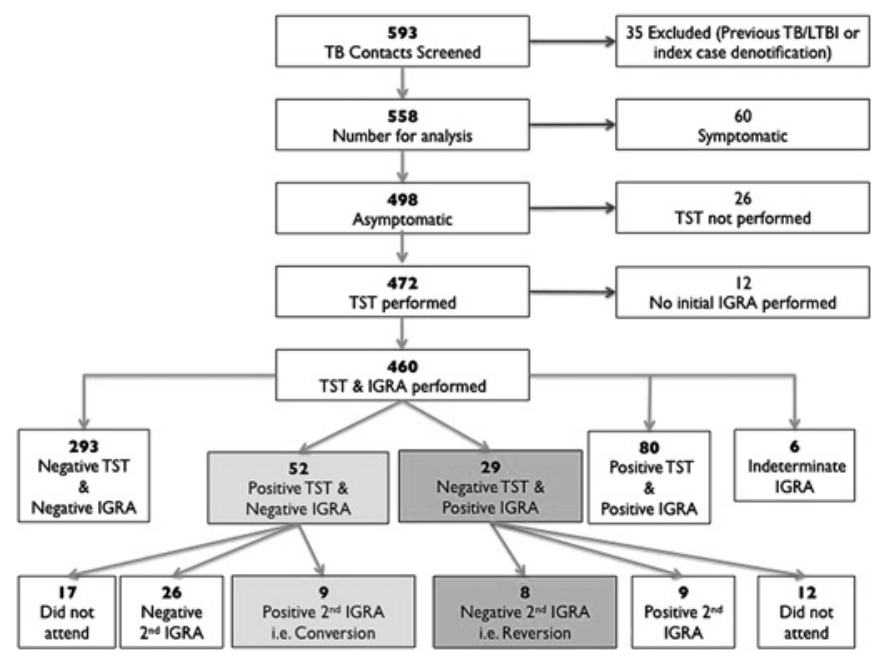

Abstract P13 Figure 1 LTBI Diagnostic Flow showing that an IGRA rescreen can both identify LTBI and reduce the number of false positives.
(17.7\%) contacts had discordant TST \& IGRA results. 52 (64\%) of these discordant cases had a positive TST \& Negative IGRA; these patients would have been discharged under NICE guidelines however, our rescreen revealed $9(17 \%)$ positive $2^{\text {nd }}$ IGRAs that is, conversion. Three of these patients were under 35 and would therefore by eligible for chemoprophylaxis. Twenty-nine (36\%) of the discordant cases had a negative TST and positive IGRA however, $8(28 \%)$ of these IGRAs reverted to negative. It is important to note that if following a single-step IGRA screening protocol (ie, without a rescreen) these cases may have been commenced on chemoprophylaxis unnecessarily (four of these reversion cases were under the age of 35).

Conclusions Our results show that adoption of either a sequential TST + ve/IGRA approach or single IGRA approach can result in a significant number of false negative LTBI diagnoses due to IGRA conversion. Conversely, we have also shown that an IGRA rescreen because of discordant TST/IGRA tests can improve LTBI diagnostic specificity and therefore reduce unnecessary chemoprophylaxis due to the effect of reversion.

\section{P14 COMPARISON OF TWO INTERFERON-GAMMA RELEASE ASSAYS (QUANTIFERON-TB GOLD IN-TUBE AND T-SPOT.TB) IN SCREENING FOR LATENT TUBERCULOSIS INFECTION (LTBI) AMONG HIV-INFECTED ADULTS ATTENDING AN INNER LONDON HIV CLINIC}

doi:10.1136/thoraxjnl-2011-201054c.14

${ }^{1} \mathrm{R}$ F Miller, ${ }^{2} \mathrm{~T}$ Mahungu, ${ }^{1} \mathrm{M}$ Young, ${ }^{1} \mathrm{D}$ Mercey, ${ }^{2} \mathrm{~S}$ Morris-Jones, ${ }^{1} \mathrm{P}$ Benn. ${ }^{1}$ Mortimer Market Centre, Camden Provider Services, Central and North West London NHS Foundation Trust, London, UK; ${ }^{2}$ Department of Microbiology, University College London Hospitals NHS Foundation Trust, London, UK

Background There is a paucity of data on the performance of interferon-gamma release assays (IGRAs) in screening HIV-infected patients for latent tuberculosis infection (LTBI).

Methods Prospective evaluation of two IGRAs [QuantiFERON-TB Gold In-Tube (QuantiFERON) and T-SPOT.TB] for screening HIVinfected patients attending an inner city clinic with a high local prevalence of tuberculosis (TB). For each patient we recorded their gender, ethnicity, country of birth, BCG status and prior treatment for TB, CD4 count, plasma HIV load and receipt of antiretroviral therapy (ART). All patients had both IGRAs (results = positive, negative, or indeterminate) and a chest radiograph.

Results Of 117 patients, 91 (78\%) were men, 48 (41\%) were white British, 29 (24.8\%) black African, 21 (18\%) European, 7 (6\%) South American and $12(10 \%)$ from other ethnic groups. Individuals born outside the UK had been resident in the UK for a median of 10 years (range 3-45). 91/117 (78\%) had previously received BCG and $14 / 117(12 \%)$ had been treated for TB a median of 9.5 years ( range 3-13) previously. Of 106 (90.6\%) receiving ART, 105 (99\%) had an undetectable plasma HIV load) and the median CD4 count was 550 (range 30-1250). Among those not receiving ART the median (range) plasma HIV load and CD4 count was 21000 copies/ $\mathrm{ml}(590-160000)$ and 520 (250-950) respectively. IGRA results are shown in the Abstract P14 table 1. Overall there was good concordance $(92.3 \%)$ between the two IGRAs. Among 14 patients previously treated for TB, $10(71.4 \%)$ had previously received BCG; 4/14 (28.6\%) had a positive IGRA (3/4 both IGRAs were positive, 1) 4 QuantiFERON was positive). Of those previously treated for TB with negative IGRAs $(\mathrm{n}=10)$ all had an undetectable HIV viral load, 9/10 had been on ART $>2$ years and median CD4 count was 670 (range 180-1250). Five other patients with CD4 $<200$ had negative IGRAs; all had previously received BCG. Mantoux testing, done in 2 of the 5 , was negative. 